\title{
The Impact of Sex Work Interruption on Blood-Derived T Cells in Sex Workers from Nairobi, Kenya
}

\author{
Kenneth Omollo,' Geneviève Boily-Larouche, Julie Lajoie,,2 Makobu Kimani, Julianna Cheruiyot, ${ }^{3}$ \\ Joshua Kimani, ${ }^{1,3}$ Julius Oyugi, ${ }^{1,3}$ and Keith Raymond Fowke ${ }^{1,2,4}$
}

\begin{abstract}
Background: Unprotected sexual intercourse exposes the female genital tract (FGT) to semen-derived antigens, which leads to a proinflammatory response. Studies have shown that this postcoital inflammatory response can lead to recruitment of activated T cells to the FGT, thereby increasing risk of HIV infection.

Objective: The purpose of this study was to evaluate the impact of sex work on activation and memory phenotypes of peripheral T cells among female sex workers (FSW) from Nairobi, Kenya.

Subjects: Thirty FSW were recruited from the Pumwani Sex Workers Cohort, 10 in each of the following groups: HIVexposed seronegative (at least 7 years in active sex work), HIV positive, and New Negative (HIV negative, less than 3 years in active sex work). Blood was obtained at three different phases (active sex work, abstinence from sex work-sex break, and following resumption of sex work). Peripheral blood mononuclear cells were isolated and stained for phenotypic markers (CD3, CD4, CD8, and CD161), memory phenotype markers (CD45RA and CCR7), activation markers (CD69, HLA-DR, and CD95), and the HIV coreceptor (CCR5). T-cell populations were compared between groups.

Results: In HIV-positive women, CD8+CCR5+ T cells declined at the sex break period, while CD4+CD161+ T cells increased when returning to sex work. All groups showed no significant changes in systemic T-cell activation markers following the interruption of sex work, however, significant reductions in naive CD8+ T cells were noted. For each of the study points, HIV positives had higher effector memory and CD8+CD95+ T cells and lower naive CD8+ T cells than the HIV-uninfected groups.

Conclusions: Interruption of sex work had subtle effects on systemic T-cell memory phenotypes.
\end{abstract}

Keywords: HIV, sex work, T cell memory, immune activation, HIV-exposed seronegative, peripheral blood mononuclear cells

\section{Introduction}

$\mathbf{U}$ NPROTECTED VAGINAL INTERCOURSE is the main mode of HIV acquisition in women. It also results in exposure to client-derived immune and sperm cells, which have a strong immune stimulatory capacity. ${ }^{1}$ Exposure to seminal fluid can also cause the release of chemokines, cytokines, and other innate factors at the female genital tract (FGT). ${ }^{2} \mathrm{~A}$ release of interleukin (IL)-8 and IL-10 has been observed in cervical explants exposed to seminal plasma. ${ }^{3}$ Recruitment of macrophages, dendritic cells, and granulocytes into the endometrium of mice has been observed within hours of mat- ing. ${ }^{4}$ In humans, Sharkey et al. reported, following exposure to seminal fluid, changes in expression of proinflammatory cytokines and chemokines and a robust recruitment of macrophages, dendritic cells, and memory T cells. ${ }^{5}$ The induction of cytokines/chemokines and the increased number of CD4+ $\mathrm{T}$ cells that is observed in the FGT after sexual activity may increase the risk of HIV acquisition in women. Sperm antigens can also induce expression of Th17 cells, ${ }^{6}$ a subset of $\mathrm{T}$ cells that are preferential targets of HIV infection. ${ }^{7}$

Susceptibility to HIV could, therefore, be influenced by how an individual responds to sex-induced T-cell activation. Differences in the susceptibility to HIV infection have been

\footnotetext{
${ }^{1}$ Department of Medical Microbiology, University of Nairobi, Nairobi, Kenya.

${ }^{2}$ Department of Medical Microbiology, University of Manitoba, Winnipeg, Manitoba, Canada.

${ }^{3}$ Kenyan AIDS Control Program, University of Nairobi, Nairobi, Kenya.

${ }^{4}$ Department of Community Health Science, University of Manitoba, Winnipeg, Manitoba, Canada.
}

(c) Kenneth Omollo, et al., 2016; Published by Mary Ann Liebert, Inc. This Open Access article is distributed under the terms of the Creative Commons Attribution Noncommercial License (http://creativecommons.org/licenses/by-nc/4.0/) which permits any noncommercial use, distribution, and reproduction in any medium, provided the original author(s) and the source are credited. 
observed between individuals. Indeed, some individuals, despite being at high risk of HIV acquisition, remain HIV uninfected [HIV-exposed seronegative (HESN)]. Both genetic and immunological factors have been identified as correlates of natural protection from HIV. ${ }^{8}$ HESN female sex workers (FSW) from Nairobi, Kenya, have been shown to have lower mucosal and peripheral immune activation than HIV-uninfected women newly practicing sex work and lowrisk women not involved in sex work, resulting in a potential reduction of targets cells at the portal of entry. ${ }^{9,10}$ In peripheral blood, HESN express low levels of the acute activation marker CD69 on T cells, ${ }^{11}$ lower expression of genes encoding T-cell immune function, ${ }^{12}$ lower production of proinflammatory cytokines, ${ }^{9}$ and reduced IL-17 and IL-22 production by Th17 cells. ${ }^{13}$ HESN sex workers also have elevated mean percentages of T-regulatory (T-reg) cells, ${ }^{11}$ leading to an immune quiescence phenotype.

A recent study from our group has shown that duration of sex work correlates with lower levels of proinflammatory cytokines and chemokines and lower frequency of CCR5+CD4+ $\mathrm{T}$ cells and activated CD8+ $\mathrm{T}$ cells in the FGT among FSWs when compared to low-risk women from the same community. ${ }^{14}$ These reductions in immune activation markers were noted as early as 1 year following initiation of sex work and were even more pronounced in women reporting being active in sex work for more than 7 years. ${ }^{14}$ Hence, it may be possible that among experienced sex workers there is an enrichment of women with low immune activation or alternatively that continuous exposure to sex antigens may induce a T-cell tolerance resulting in reduced immune activation. What is not known is the duration of the sex-induced reduction in immune activation and its potential impact on HIV susceptibility. This is particularly important as a break from sex work has previously been associated with late seroconversion in some HESN FSWs from the Pumwani cohort. ${ }^{15}$

Based on these observations, we hypothesized that HESN may have better control of sex-induced immune activation, by a yet unknown mechanism, compared to those that have been in sex work for a shorter period. This study was designed to evaluate the impact of sex work on systemic T cells. We compared the response to interruption of sex work among HESN, New Negative, and HIV-positive sex workers to assess difference in immune activation among the groups.

\section{Materials and Methods}

\section{Study participants}

This study was conducted among FSWs from the Pumwani Sex Workers Cohort. This cohort was established in 1985 and has been well described. ${ }^{16-18}$ Thirty women were followed for this study with 10 women included in each group; HESN, New Negatives, and HIV positive. Based on an epidemiological model, ${ }^{17}$ HESN were women who were actively involved in sex work for over 7 years and remained seronegative for HIV. New Negatives were women who had been in sex work for 3 years or less and HIV uninfected. HIVpositive women were those with confirmed HIV serology, had CD4 counts above 350 cells $/ \mathrm{mm}^{3}$, and were antiretroviral therapy naive in accordance with Kenyan treatment guidelines at the time of the study. Women were included if they were 18 years or older and if they reported being actively involved in sex work. Women were excluded if they were pregnant, breastfeeding, in menopause, or had any sexually transmitted infections at recruitment. At every study visit, each participant answered a questionnaire regarding sociodemographics, sexual and reproductive history, number of clients and regular partners, and other clinical factors. The Kenyatta National Hospital/University of Nairobi Ethics Review Committee and the University of Manitoba Ethics Review Board approved this study. Written informed consent was obtained from each participant.

\section{Experimental design}

This was a substudy nested within the on-going Longitudinal Assessment of Mucosal Immune Quiescence (LAMIQ) study. Blood samples were collected at three time points over a 3-month period. Visit 1 constituted the baseline sample during active sex work. Visit 2 samples were taken during a 4-week sex break when participants were asked to abstain from sexual activities. Women were compensated for the loss of income during the abstinence period. Abstinence was confirmed using a prostate-specific antigen (PSA) rapid test kit (Seratec PSA Semiquant, Göttingen, Germany). Following the sex break period, the women then went back to active sex work. Samples taken at Visit 3 were collected after reintroduction of sex work. To synchronize the time of sampling among participants, all study samples were taken within 10 days of the completion of menses, which constituted the follicular phase of the menstrual cycle.

\section{Sample collection}

At each visit, $20 \mathrm{ml}$ of venous blood was collected by venipuncture into sodium heparin anticoagulant tubes. Peripheral blood mononuclear cells (PBMC) were then isolated using the Ficoll-Hypaque technique.

\section{Immunophenotyping assay}

Fresh PBMCs were stained with a cocktail of 10 fluorochrome-conjugated monoclonal antibodies from Beckton Dickinson (BD) Biosciences (Mississauga, Canada): Anti-CD3PECy5, Anti-CD4-FITC, Anti-CD8-V500, Anti-CD45RAAlexa700, Anti-HLA-DR-APC-H7, Anti-CD69-PECy7, Anti-CCR7-PE-CF594, Anti-CCR5-V450, Anti-CD161-APC, and Anti-CD95-PE. The cells were then incubated for $30 \mathrm{~min}$ at $4^{\circ} \mathrm{C}$ and then washed with $100 \mu \mathrm{l}$ of FACS Buffer by centrifuging at $1,600 \mathrm{rpm}$ for $10 \mathrm{~min}$ at $4{ }^{\circ} \mathrm{C}$. The supernatant was then removed. The stained cells were then resuspended in $300 \mu \mathrm{l}$ of Fix solution (1\% Paraformaldehyde: one part of BD Fix solution and three parts of FACS buffer) before acquisition on the BD LSR II cytometer. Expression of these markers was analyzed using FlowJO software (version 10.0.7; Tree Star, Ashland, OR). Memory markers were defined as follows: Naive cells CD45RA+ CCR7+, terminally differentiated $\mathrm{T}$ effector cells CD45RA+ CCR7-, T central memory CD45RA- CCR7+, and T effector memory CD45RACCR7-. An example of the flow cytometry gating is provided in Figure 1.

\section{Statistical analyses}

Statistical analyses were performed using GraphPad Prism software (version 6.0; La Jolla, CA). Gaussian distribution was tested by the D'agostino and Pearson Omnibus test. The 


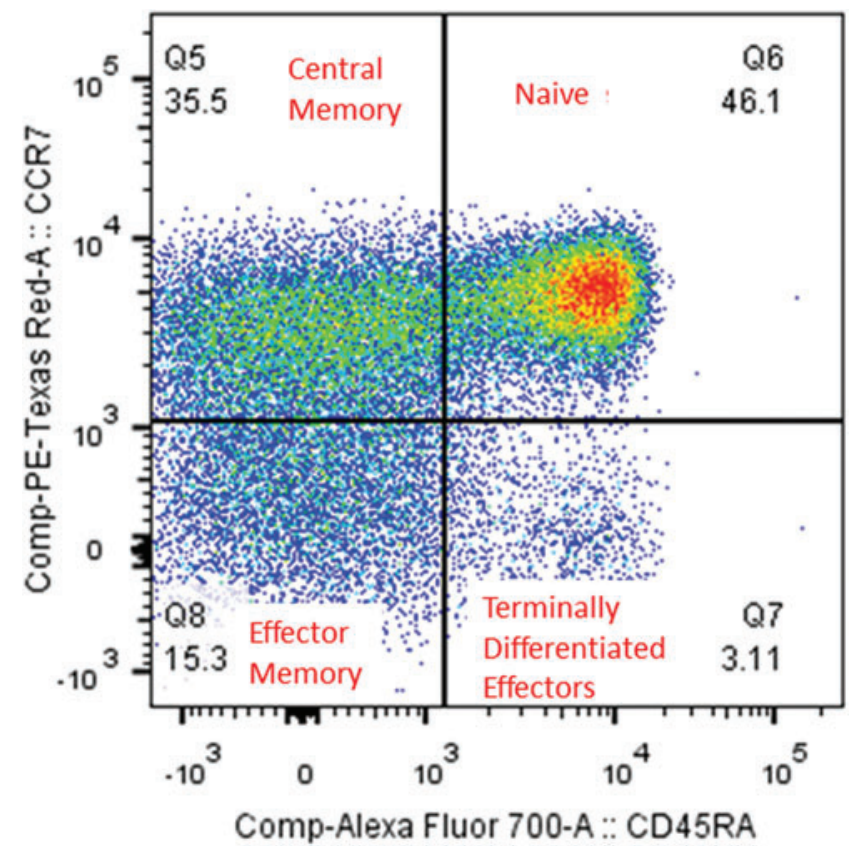

FIG. 1. Gating strategy on T-cell memory phenotypes. T-cell memory phenotypes were described based on CCR7 and CD45RA expression as effector memory $\left(\mathrm{T}_{\mathrm{EM}}\right)$; naive $\left(\mathrm{T}_{\text {naive }}\right)$; central memory $\left(\mathrm{T}_{\mathrm{CM}}\right)$, and terminally differentiated effector $\mathrm{T}$ cells $\left(\mathrm{T}_{\text {eff }}\right)$. Color images available online at www.liebertpub.com/aid
Kruskal-Wallis test was used to compare variables that were not normally distributed. One-way ANOVA was used to test mean differences of normally distributed variables. Comparison of mean expression of activation markers between time points was done using the Tukey's multiple comparison test. Comparison of mean density of expression of activation and memory markers between the three groups and time point was done using two-way ANOVA, and if statistically significant, the Dunn's multiple comparison test was used as the post-test. The chisquare test was used to compare categorical variables. Statistical significance was accepted if the $p$-value was $\leq .05$.

\section{Results}

\section{Sociodemographics}

We recruited 30 women from the Pumwani Sex Worker Cohort in this study, 10 in each group. Table 1 shows that there was no difference in age between the three groups. Due to the criterion used to define the length of follow-up for HESN and New Negatives, there was a significant difference in the duration of sex work for the three groups with the HESN having the highest median duration of 11.5 years [interquartile range (IQR) 9.5-13.75], New Negatives 3.0 and HIV positives 7.0 (Table 1). CD4 counts were done for all participants at recruitment, and as expected, the HIVinfected women had the lowest median count of 610 cells/ $\mathrm{mm}^{3}$ [IQR 545-745], while the HESN and New Negatives had median CD4 counts of 1,011 and 1,033, respectively. The HIV-positive group had more time (in days) from last menses

Table 1. Sociodemographic and Clinical Characteristics of Study Participants

\begin{tabular}{|c|c|c|c|c|}
\hline & $\begin{array}{l}H E S N \\
\mathrm{n}=10\end{array}$ & $\begin{array}{c}\text { New Negative } \\
\mathrm{n}=10\end{array}$ & $\begin{array}{l}\text { HIV-Positive } \\
\mathrm{n}=10\end{array}$ & $\mathrm{p}$-value \\
\hline Age & $37.5(34.5-44.25)$ & $34.5(32-39.5)$ & $38(34-45.25)$ & 0.47 \\
\hline Duration of Sex Work (years) & $11.5(9.5-13.75)$ & $3(2-3)$ & $7(3-12)$ & $<0.01$ \\
\hline $\begin{array}{l}\text { CD4 Count at Recruitment } \\
(\text { cells } / \mu \mathrm{l})\end{array}$ & $1011(800-1111)$ & $1033(921.3-1193)$ & $610(545-745)$ & $<0.01$ \\
\hline \multicolumn{5}{|l|}{ Days Post- menses } \\
\hline SW: & $8.5(7-13)$ & $8(6-9)$ & $11(8-14.5)$ & 0.05 \\
\hline SB: & $10(7.25-18.25)$ & $9(5.75-25.25)$ & $12.5(8-19.25)$ & 0.83 \\
\hline RSW: & $19(9.75-23.25)$ & $7.25(10-23)$ & $7.5(4.75-15)$ & 0.09 \\
\hline \multicolumn{5}{|l|}{ No. of clients in the last 7 days } \\
\hline SW: & $5(2.75-18)$ & $2(0.75-7.25)$ & $12(5.75-20)$ & 0.03 \\
\hline SB: & 0 & 0 & 0 & NA \\
\hline RSW: & $4(0.75-8)$ & $2(0.5-8.5)$ & $2.5(0.75-10)$ & 0.81 \\
\hline \multicolumn{5}{|c|}{ Reported always using a condom with clients in the last 7 days } \\
\hline SW: & $7(70 \%)$ & $6(60 \%)$ & $8(80 \%)$ & 0.88 \\
\hline SB: & NA & NA & NA & \\
\hline RSW: & $8(80 \%)$ & $7(70 \%)$ & $9(90 \%)$ & 0.33 \\
\hline \multicolumn{5}{|c|}{ Reported having a regular sex partner } \\
\hline SW: & $5(50 \%)$ & $7(70 \%)$ & $5(50 \%)$ & 0.58 \\
\hline SB: & $5(50 \%)$ & $5(50 \%)$ & $5(50 \%)$ & 1.00 \\
\hline RSW: & $5(50 \%)$ & $4(40 \%)$ & $4(40 \%)$ & 0.87 \\
\hline \multicolumn{5}{|c|}{ Reported unprotected sex with a client/partner in the last 2 weeks } \\
\hline SW: & $5(50 \%)$ & $4(40 \%)$ & $5(50 \%)$ & 0.87 \\
\hline SB: & $0(0 \%)$ & $0(0 \%)$ & $0(0 \%)$ & NA \\
\hline RSW: & $3(30 \%)$ & $2(20 \%)$ & $1(10 \%)$ & 0.53 \\
\hline $\begin{array}{l}\text { PSA Test Results during the } \\
\text { sex break period }\end{array}$ & $2(20 \%)$ & $0(0 \%)$ & $0(0 \%)$ & 0.13 \\
\hline
\end{tabular}

Data are median (interquartile range) and mean (standard deviation).

NA, not applicable; NEG, negative; POS, positive; RSW, return to sex work; SB, sex break; SW, sex work. 
to sampling at visit 1 compared to the HESN and New Negatives $(11,8.5,8$, respectively, $p=.05)$, however, all of these times would have been in the follicular phase of the menstrual cycle. The HIV-positive group also had significantly higher median number of clients in the week before the first study visit $(p<.03)$ compared to HESN and New Negative women (12; $5 ; 2$; respectively). No differences were observed among the three groups for number of regular partners, condom use with either clients or regular partners, and PSA test positivity. However, PSA was detected in two HESN women despite the fact that they reported abstinence during the sex break.

\section{Comparison of T-cell activation marker expression}

To assess the impact of sex work on peripheral immune activation, we measured the density of expression of activation markers on $\mathrm{T}$-cell subsets. T cell activation was defined by the single expression of CCR5, CD95, CD69, or HLA-DR on CD4+ and CD8+ T cells. No difference in expression of these markers could be observed between active sex work, sex break, and return to sex work within the groups in the CD4+ Tcell population. In the HIV-positive group, in the CD8+ CCR5+ subset, we observed a significant decrease from active sex work (V1) 7.5\% (IQR 5.2-9.9) to interruption of sex work (V2) 3.5\% [IQR 2.3-6.5, $(p<.01)]$ (Fig. 2A).

\section{Comparison of CD161 expression}

CD161 was used as a surrogate marker for Th17 cells in the CD4+ population and Mucosa-Associated Invariant $\mathrm{T}$ cells (MAIT) in the CD8+ population. Th17 cells are important HIV target cells and have been showed to be quickly depleted in HIV infected. ${ }^{19}$ MAIT cells play an important role in host defense against pathogens and despite not being direct HIV target cells, this population is depleted in patients with early infection, which could increase their susceptibility to opportunistic infections. ${ }^{20} \mathrm{We}$ analyzed expression of CD161 to determine if these cell populations are affected by an interruption of sex work. In the HIVpositive group, a significant increase in CD161 expression was observed in the CD4+ subset between sex break $8.9 \%$ (IQR 4.0-13.9) and upon return to sex work 13.0\% [IQR 9.6-17.0), $p=.05$; Fig. 2B].

\section{T-cell memory phenotypes}

An increase in the proportion of terminally differentiated effector $\left(\mathrm{T}_{\mathrm{EFF}}\right) \mathrm{CD} 4+\mathrm{T}$-cell subset was observed in the HIVpositive group during the sex break (2.1\%, IQR 1.3-3.4), followed by a reduction upon resumption of sex work $(0.8 \%$, IQR 0.5-3.4; ANOVA, $p=.04$ ) (Fig. 2C). Furthermore, a trend toward decreasing proportion of naive CD8+ $\mathrm{T}$ cells over the three time points in the New Negative group was observed (Fig. 2D; $p=.09$ ).

\section{Comparison of pattern of response to interruption of sex work}

No significant differences were observed between the three groups during the sex break period in terms of expression of activation markers on both CD4+ and CD8+ T cells (data not shown). Interestingly, independent of groups, interruption of sex work reduced the proportion of CD8+ naive $\mathrm{T}$ cells [Fig. 3B, Time (SW/SB/RSW) ANOVA $p=.04$ ].

\section{Impact of HIV on memory T cells and activation markers}

Previous studies have shown that HIV infection results in modifications of the activation status and memory phenotype
FIG. 2. T-cell populations at time points. (A) A decrease in the CCR5 expressing CD8+ $\mathrm{T}$ cells in HIV-positive sex workers during the sex break. (B) Increased CD161 expression on CD4+ T cells in HIVpositive sex workers upon resumption of sex work. (C) Increased proportion of $\mathrm{CD} 4+$ terminally differentiated effector $\mathrm{T}$ cells in HIV-positive participants during the sex break. (D) Declining frequency of CD8+-naive T cells in New Negative participants over time.
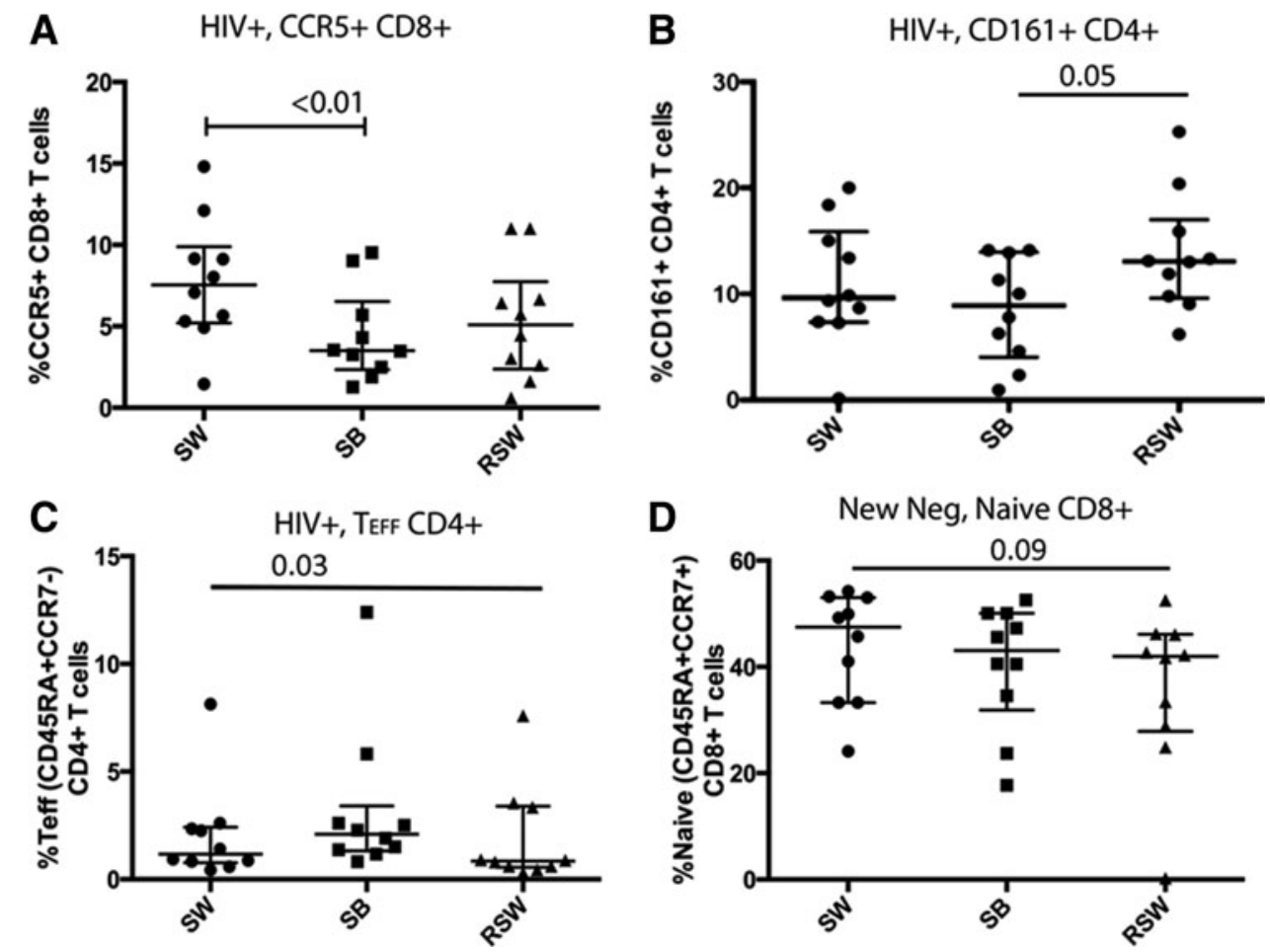

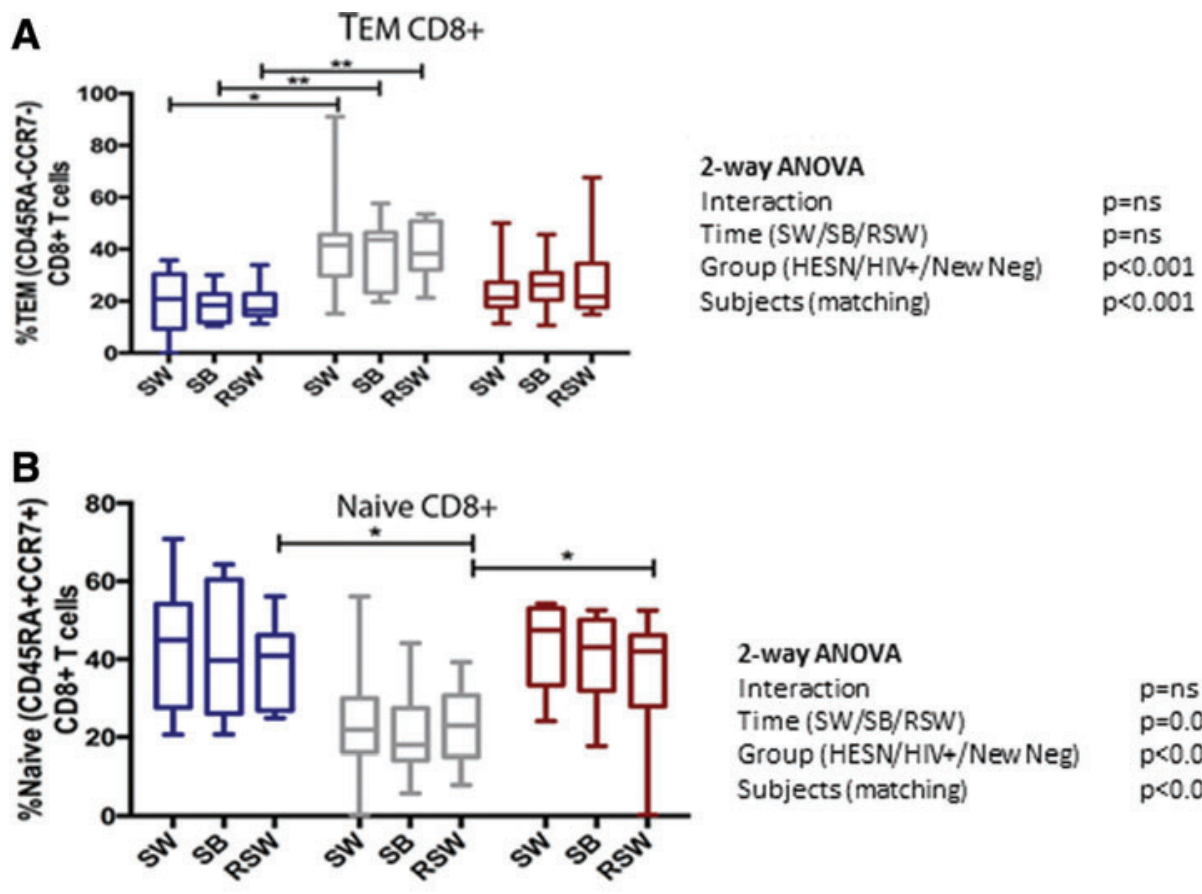

\section{2-way ANOVA}

Interaction

Time (SW/SB/RSW)

Group (HESN/HIV+/New Neg)

Subjects (matching)

\section{2-way ANOVA}

Interaction

Time (SW/SB/RSW)

Group (HESN/HIV+/New Neg)

Subjects (matching) $\quad \mathrm{p}<0.001$
FIG. 3. Proportions of effector memory, naive, and CD95+ CD8+ T cells between groups. HIV-positive sex workers had a higher proportion of effector memory (A) and CD95+ CD8+ T cells (C), but lower naive $\mathrm{CD} 8+\mathrm{T}$ cells $(\mathbf{B})$ than HESN and New Negatives. Independent of groups, sex work had an impact on CD8+-naive $\mathrm{T}$ cells (C). HESN, HIV-exposed seronegative. Color images available online at www.liebertpub .com/aid

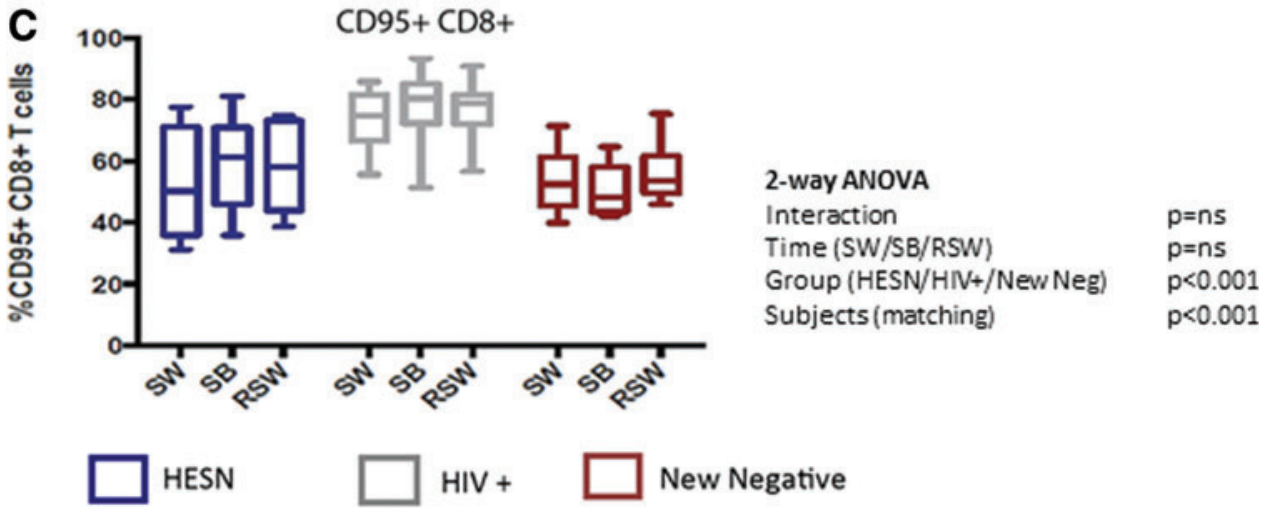

of T cells. We observed that relative to the HESN and New Negative groups, HIV-positive FSWs had a higher proportion of CD8+ effector memory $\mathrm{T}$ cells $\left(\mathrm{T}_{\mathrm{EM}}\right)$ (Fig. 3A Groups ANOVA $p<.001$ ), lower proportion of naive CD8+ T cells (Fig. 3B Groups ANOVA $p<.01$ ), and higher proportion of $\mathrm{CD} 8+\mathrm{T}$ cells that expressed the activation/apoptosis marker CD95 (Fig. 3C Groups ANOVA $p<.001$ ).

\section{Discussion}

Exposure of the FGT to semen-derived antigens during unprotected vaginal intercourse can result in an inflammatory response leading to recruitment of $\mathrm{T}$ cells to the mucosa, ${ }^{5}$ which can increase the risk of HIV infection. Sex antigen stimulation may also lead to the expansion and differentiation of memory $\mathrm{T}$ cells that are primed to mount secondary responses against these antigens upon reexposure. We have previously reported that HESN have higher levels of systemic regulatory $\mathrm{T}$ cells (Tregs) and lower levels of systemic immune activation. ${ }^{11}$ In this study, we hypothesize that sex work results in exposure to immune stimulants, which can drive systemic activation of $\mathrm{T}$ cells, and based on HESN
FSW-reported higher levels of systemic Tregs and lower immune activation state, they would be able to downregulate such sex-driven immune activation more effectively than their New Negative and HIV-positive counterparts.

We observed that following interruption of sex work, fewer CD8+ T cells expressed CCR5 in HIV-positive FSWs (Fig. 2A), whereas the proportion of effector and memory $\mathrm{T}$ cells did not change in this subset. CCR5 is a chemokine receptor, which plays a critical role in the migration of antigen-specific effector and memory CD8+ T cells to tissues and secondary lymphoid organs. ${ }^{21}$ This suggests that in the HIV-positive women, taking a break from sex work may reduce sex-induced inflammation at the FGT, and this is reflected in the systemic compartment through a reduction of migratory capacity of memory CD8+ $\mathrm{T}$ cells to the FGT. Notably, even in the context of HIV-induced immune activation, an interruption of commercial sexual activity reduces peripheral expression of CCR5 on CD8+ T cells. This underlines the strong impact of sex-related immune stimulation. We further observed that compared to HESN and New Negative, HIV-positive sex workers reported a higher number of clients in the week before starting the study (Visit 1). Despite 
the fact that the reported unprotected sex in this period was similar in all groups, the HIV-positive women could still have had higher exposure to seminal fluids while in active sex work leading to inflammation and therefore explaining, in part, why the interruption of sex work has a greater impact on CCR5 expression on T cells in the HIV-positive group. It may also be possible that interruption of sex work has a greater effect on the HIV-positive women because of preexisting immune dysregulation on account of HIV infection.

CD4+ T cells expressing CD161 are considered to be Th17 cells, ${ }^{22}$ a proinflammatory T-cell subset. Th17 cells play a role in mucosal antimicrobial immune responses. ${ }^{23}$ These cells have been shown to be preferentially infected by HIV and depleted from the gut ${ }^{19}$ and cervix ${ }^{24}$ of HIV-infected individuals. In the context of sex work, we observed that HIVpositive sex workers displayed a significant increase $(p=.05)$ in the proportion of Th17 cells in the blood following resumption of sex work (Fig. 2B). As self-reported condom use was almost $100 \%$ during this period, it is hard to draw a conclusion on this observation. However, it has been shown that self-reported use of condom does not always match with the reality, ${ }^{25}$ which might have been the case herein.

Overall, we observed that an interruption in sex work did not affect systemic expression of markers of T-cell activation, the frequency of Th17 cells, and memory T-cell phenotypes in HESN and New Negatives. However, we observed a trend $(p=.09)$ in decreasing frequency of naive CD8+ T cells in the blood over time among New Negative women (Fig. 2D). This could imply that these women, who are relatively new in sex work, develop a specific memory against sex antigens over time, decreasing their pool of naive T cells. Although we did not observe a concomitant increase in the frequency of the other memory phenotypes in blood, it is possible that these may populate the secondary lymphoid organs to be differentiated into effector cells capable of migrating to the FGT to perform their effector functions. Furthermore, in the HIV-positive group, we observed an increased frequency of terminally differentiated CD4+ T cells implying that the absence of sex-driven antigenic stimulation at the genital tract results in the cells trafficking back into the blood (Fig. 2C).

An important observation made in this study is that, independent of sexual activity, HIV-positive participants exhibited higher levels of CD8+ effector memory $\mathrm{T}$ cell and higher CD95 expressing CD8+ T cells (Fig. 3A,C) that are consistent with previous studies. Alterations in the frequencies of $\mathrm{CD} 8+$ naive and $\mathrm{T}_{\mathrm{EM}}$ during the course of HIV infection have been described ${ }^{26}$ with an expanded $\mathrm{T}_{\text {EM }}$ cell population $^{27,28}$ and lower frequency of naive T cells ${ }^{29}$ compared to healthy controls or acutely infected persons. This skewed distribution of memory phenotypes has been associated with immune activation and disease progression. ${ }^{30}$ The increased expression of CD95 on CD8+ T cells is also a feature of HIV infection ${ }^{31,32}$ and is evidence of increased activation or apoptotic potential of memory $\mathrm{T}$ cells. Taken together, these observations demonstrate that our assay, which detects ex vivo T-cell phenotypes and activation, is consistent with other studies.

The impact of the menstrual cycle on immune activation may be an important factor when studying the immune system in women. To control for this, we collected samples at the follicular phase of the menstrual cycle, although HIV-positive women did have more time from last menses to the first visit (11 days vs. 8.5 and 8 for HESN and New Negatives).

Condom use is also an important cofactor to consider in this study. We did not observe any significant differences in self-reported condom use with clients or unprotected sex with regular partners among the three groups. This implies that instances of protected and unprotected sex during the phases of active sex work were similar across groups. However, we did not test for PSA at all study visits and this presents a limitation in our ability to predict exposure to seminal fluid during sex work. We acknowledge that this may impact the results presented herein. Sharkey et al. reported that leukocyte recruitment to the FGT did not occur with abstinence or condom use, concluding that direct interaction between seminal constituents and genital cells is responsible for the inflammatory response. ${ }^{5}$ Therefore, condom use or the physical stimulation associated with sexual intercourse may not be responsible for the induction of inflammation at the genital tract or in peripheral blood.

We were unable to demonstrate any significant difference in the systemic levels of T-cell immune activation in response to the interruption of sex work across all groups. The modest sample size of this study (10 participants per group) may be a limitation in our ability to detect a difference between the groups. It is also possible that the impact of sex work on T-cell activation remains local with activation and proliferation of resident genital mucosal $\mathrm{T}$-cell population that do not traffic back to the blood compartment. Further studies looking at genital T-cell activation are needed to address this question.

Importantly, this study did demonstrate that in all three groups, the initiation of a period of sexual abstinence resulted in a significant decrease in the proportion of systemic CD8+ T cells, which were of a naive phenotype (Fig. 3B). This may suggest that any immune regulation that was initiated because of sex work may be reduced during the sex break period, thereby allowing more naive cells to differentiate into various memory and effector phenotypes. A careful investigation of Treg mechanisms in the systemic and mucosal compartments during sex work is needed.

Overall, this study showed that sex work does have a significant impact on the systemic T-cell memory phenotypes, although changes in T-cell activation markers were not noted. This study suggests that additional investigations on the impact of sexual activity on the local and systemic immune response are warranted.

\section{Acknowledgments}

Our gratitude goes to all the staff and clients of the Majengo Clinic and technicians at the Kenya AIDS Control Program, University of Nairobi.

\section{Author Disclosure Statement}

No competing financial interests exist.

\section{References}

1. Via CS, Tsokos GC, Stocks NI, Clerici M, Shearer GM: Human in vitro allogeneic responses. Demonstration of three pathways of $\mathrm{T}$ helper cell activation. J Immunol 1990;144:2524-2528.

2. Sharkey DJ, Macpherson AM, Tremellen KP, Robertson SA: Seminal plasma differentially regulates inflammatory 
cytokine gene expression in human cervical and vaginal epithelial cells. Mol Hum Reprod 2007;13:491-501.

3. Denison FC, Grant VE, Calder AA, Kelly RW: Seminal plasma components stimulate interleukin-8 and interleukin10 release. Mol Hum Reprod 1999;5:220-226.

4. De M, Choudhuri R, Wood GW: Determination of the number and distribution of macrophages, lymphocytes, and granulocytes in the mouse uterus from mating through implantation. J Leukoc Biol 1991;50:252-262.

5. Sharkey DJ, Tremellen KP, Jasper MJ, Gemzell-Danielsson K, Robertson SA: Seminal fluid induces leukocyte recruitment and cytokine and chemokine mRNA expression in the human cervix after coitus. J Immunol 2012;188:2445-2454.

6. Lasarte S, Elsner D, Guia-Gonzalez M, Ramos-Medina R, Sanchez-Ramon S, Esponda P, et al.: Female sex hormones regulate the Th17 immune response to sperm and Candida albicans. Hum Reprod 2013;28:3283-3291.

7. Prendergast A, Prado JG, Kang YH, Chen F, Riddell LA, Luzzi G, et al.: HIV-1 infection is characterized by profound depletion of CD161+ Th17 cells and gradual decline in regulatory T cells. AIDS 2010;24:491-502.

8. Taborda-Vanegas N, Zapata W, Rugeles MT: Genetic and immunological factors involved in natural resistance to HIV-1 infection. Open Virol J 2011;5:35-43.

9. Lajoie J, Juno J, Burgener A, Rahman S, Mogk K, Wachihi C, et al.: A distinct cytokine and chemokine profile at the genital mucosa is associated with $\mathrm{HIV}-1$ protection among HIV-exposed seronegative commercial sex workers. Mucosal Immunol 2012;5:277-287.

10. Card CM, Keynan Y, Lajoie J, Bell CP, Dawood M, Becker $\mathrm{M}$, et al:: HIV controllers are distinguished by chemokine expression profile and HIV-specific T-cell proliferative potential. J Acquir Immune Defic Syndr 2012;59:427-437.

11. Card CM, McLaren PJ, Wachihi C, Kimani J, Plummer FA, Fowke KR: Decreased immune activation in resistance to HIV-1 infection is associated with an elevated frequency of CD4(+)CD25(+)FOXP3(+) regulatory T cells. J Infect Dis 2009;199:1318-1322.

12. Songok EM, Luo M, Liang B, McLaren P, Kaefer N, Apidi W, et al: : Microarray analysis of HIV resistant female sex workers reveal a gene expression signature pattern reminiscent of a lowered immune activation state. PLoS One 2012;7:e30048.

13. Chege D, Chai Y, Huibner S, Kain T, Wachihi C, Kimani M, et al.: Blunted IL17/IL22 and pro-inflammatory cytokine responses in the genital tract and blood of HIVexposed, seronegative female sex workers in Kenya. PLoS One 2012;7:e43670.

14. Lajoie J, Kimani M, Plummer FA, Nyamiobo F, Kaul R, Kimani $\mathrm{J}$, et al:: Association of sex work with reduced activation of the mucosal immune system. J Infect Dis 2014;210:319-329.

15. Kaul R, Rowland-Jones SL, Kimani J, Dong T, Yang HB, Kiama $\mathrm{P}$, et al.: Late seroconversion in HIV-resistant Nairobi prostitutes despite pre-existing HIV-specific CD8+ responses. J Clin Investig 2001;107:341-349.

16. Koesters SA, Matu L, Kiama P, Anzala O, Embree J, Plummer FA, et al:: Elevation of immune activation in kenyan women is associated with alterations in immune function: Implications for vaccine development. J Clin Immunol 2004;24:702-709.

17. Fowke KR, Nagelkerke NJ, Kimani J, Simonsen JN, Anzala AO, Bwayo JJ, et al.: Resistance to HIV-1 infection among persistently seronegative prostitutes in Nairobi, Kenya. Lancet 1996;348:1347-1351.
18. Horton RE, McLaren PJ, Fowke K, Kimani J, Ball TB: Cohorts for the study of HIV-1-exposed but uninfected individuals: Benefits and limitations. J Infect Dis 2010; 202 Suppl 3:S377-S381.

19. Brenchley JM, Paiardini M, Knox KS, Asher AI, Cervasi B, Asher TE, et al.: Differential Th17 CD4 T-cell depletion in pathogenic and nonpathogenic lentiviral infections. Blood 2008;112:2826-2835.

20. Cosgrove C, Ussher JE, Rauch A, Gartner K, Kurioka A, Huhn MH, et al:: Early and nonreversible decrease of CD161+// MAIT cells in HIV infection. Blood 2013;121:951-961.

21. Fukada K, Sobao Y, Tomiyama H, Oka S, Takiguchi M: Functional expression of the chemokine receptor CCR5 on virus epitope-specific memory and effector CD8+ T cells. J Immunol 2002;168:2225-2232.

22. Fergusson JR, Fleming VM, Klenerman P: CD161expressing human T cells. Front Immunol 2011;2:36.

23. Dandekar S, George MD, Baumler AJ: Th17 cells, HIV and the gut mucosal barrier. Curr Opin HIV AIDS 2010;5:173-178.

24. McKinnon LR, Nyanga B, Chege D, Izulla P, Kimani M, Huibner $\mathrm{S}$, et al.: Characterization of a human cervical CD4+ T cell subset coexpressing multiple markers of HIV susceptibility. J Immunol 2011;187:6032-6042.

25. Zimmerman RS, Morisky DE, Harrison L, Mark HD: Validity of behavioral measures as proxies for HIV-related outcomes. J Acquir Immune Defic Syndr 2014;66 Suppl 3:S285-S292.

26. Seth A, Markee J, Hoering A, Sevin A, Sabath DE, Schmitz $\mathrm{JE}$, et al: Alterations in T cell phenotype and human immunodeficiency virus type $1-$ Specific cytotoxicity after potent antiretroviral therapy. J Infect Dis 2001;183:722-729.

27. Tinago W, Coghlan E, Macken A, McAndrews J, Doak B, Prior-Fuller C, et al: : Clinical, immunological and treatmentrelated factors associated with normalised CD4+/CD8+ Tcell ratio: Effect of naive and memory T-cell subsets. PLoS One 2014;9:e97011.

28. Ghiglione Y, Falivene J, Ruiz MJ, Laufer N, Socias ME, Cahn P, et al.: Early skewed distribution of total and HIVspecific CD8+ T-cell memory phenotypes during primary HIV infection is related to reduced antiviral activity and faster disease progression. PLoS One 2014;9:e104235.

29. Tiba F, Nauwelaers F, Sangare L, Coulibaly B, Krausslich HG, Bohler T: Activation and maturation of peripheral blood $\mathrm{T}$ cells in HIV-1-infected and HIV-1-uninfected adults in Burkina Faso: A cross-sectional study. J Int AIDS Soc 2011;14:57.

30. Moir S, Chun TW, Fauci AS: Pathogenic mechanisms of HIV disease. Annu Rev Pathol 2011;6:223-248.

31. Mueller YM, De Rosa SC, Hutton JA, Witek J, Roederer M, Altman JD, et al:: Increased CD95/Fas-induced apoptosis of HIV-specific CD8(+) T cells. Immunity 2001;15:871-882.

32. Wood KL, Twigg HL III, Doseff AI: Dysregulation of CD8+ lymphocyte apoptosis, chronic disease, and immune regulation. Front Biosci 2009;14:3771-3781.

Address correspondence to: Keith Raymond Fowke

Department of Medical Microbiology

University of Manitoba

745 Bannatyne, Room 539

Winnipeg R3E OJ9

Manitoba

Canada

E-mail: keith.fowke@umanitoba.ca 